\title{
Dermatitis Atópica en adultos
}

\section{Atopic Dermatitis in adults}

Pág. 69,74

Recibido: 06-08-2020

Aceptado: 04-09-2020

Dr. Luis Seas Garita 1

Dra. Maria Fernanda Ochoa Perez ${ }^{2}$

Dra. Alison Villachica Madriz ${ }^{3}$

1,2,3. Médico general, Caja Costarricense del Seguro Social, Cartago Costa Rica

\section{RESUMEN}

La dermatitis atópica o eczema, es la enfermedad cutánea crónica en recaídas más común, que afecta tanto a niños como adultos en todo el mundo y que, aparece con frecuencia en familias con otras enfermedades atópicas como el asma, la rinitis alérgica y la alergia alimentaria. Se trata de una enfermedad multifactorial que es resultante de la interacción de factores ambientales, defectos en la función barrera y una serie de factores inmunológicos. Este artículo ofrece una visión actualizada de la epidemiología, diagnóstico, tratamiento, prevención y factores que ayudan a disminuir los efectos negativos de la dermatitis atópica en la calidad de vida de los pacientes.

\section{PALABRAS CLAVE}

Dermatitis atópica, eczema atópico, adultos.

\section{ABSTRACT}

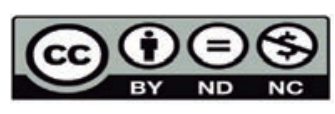

Atopic dermatitis, or eczema, is the most common chronic skin disease in relapses, affecting both children and adults worldwide and appearing frequently in families with other atopic diseases such as asthma, allergic rhinitis, and food allergy. It is a multifactorial disease that results from the interaction of environmental factors, defects in barrier function and a series of immunological factors. This article offers an updated view of the epidemiology, diagnosis, treatment, prevention and factors that help decrease the negative effects of atopic dermatitis on the quality of life of patients.

\section{KEYWORDS}

Atopic dermatitis, atopic eczema, adults

\section{INTRODUCCIÓN}

La dermatitis atópica (DA) es una dermatosis inflamatoria de curso crónico, caracterizada por un intenso prurito y en la que los pacientes suelen asociar antecedentes personales 0 familiares de atopia. La prevalencia de la DA en 
niños se estima entre un $10-20 \%$ y en adultos entre $1-3 \%$ (1). Su alta prevalencia en la edad pediátrica hace que en ocasiones se subestime el impacto de la enfermedad en edad adulta.

Los adultos con dermatitis atópica tienen una forma persistente en la infancia o con inicio después de los 18 años $\left(2^{*}\right)$. A pesar de que la mayoría de los casos en adultos corresponden a quienes la enfermedad se les presentó en su infancia, pero permanece en su vida adulta, también existe un reducido porcentaje de personas, quienes presentaron eczema atópico por primera vez en edad adulta.

La cronicidad y visibilidad de la enfermedad, junto con síntomas como picazón severa, daño en la piel, dolor y pérdida de sueño pueden tener un efecto profundo sobre la calidad de vida de los pacientes y sus familias (3). Además, el deterioro físico y psicológico que conlleva puede dificultar las relaciones con los demás y conducir al aislamiento social. La presentación clínica de $A D$ incluye prurito, xerosis y lesiones eccematosas, y su patología se caracteriza por interacciones entre defectos de la barrera cutánea y desregulación inmune, con evidencia reciente que sugiere que es un trastorno sistémico (4).

El objetivo de esta revisión es ofrecer una visión actualizada sobre el diagnóstico, tratamiento y otros aspectos relevantes de la dermatitis atópica en adultos, abordando también la orientación precoz de los pacientes y familiares.

\section{MÉTODO}

Se realiza una revisión bibliográfica de los últimos 5 años, principalmente en las bases de datos PubMed y Scielo sobre el tema, tanto en español como en inglés. Además, se abordan aspectos de interés relacionados con las manifestaciones clínicas, epidemiologíia, criterios diagnósticos y tratamiento. Los métodos de búsqueda se obtienenuvieron a través de la Biblioteca Nacional de Salud y Seguridad Social (BINASSS) y el Sistema de Bibliotecas, documentación e información de la Universidad de Costa Rica (SIBDI). Como parte de la revisión, se excluyefueron excluidos todos los artículos cuya fecha de publicación sobre pasara los cinco años de antigüedad y que no contengacontuvieran información relevante para el objetivo del artículo. Los criterios de inclusión se basan en los resultados de las búsquedas en las diferentes bases de datos: "dermatitis atópica", "prurito", "alergia en piel", artículos en español e inglés, principalmente en población adulta.

\section{EPIDEMIOLOGÍA}

La prevalencia de DA ha aumentado en todo el mundo a lo largo de los últimos 30 años (5), a tal punto que ahora es una de las enfermedades crónicas más comunes, que afectan a aproximadamente a un quinto de la población en países desarrollados. Es una enfermedad que presenta predilección por la etnia blanca y aquellos que habitan en entornos urbanos, siendo la prevalencia menor en zonas rurales (6).

La prevalencia se ha duplicado o triplicado en países industrializados en las últimas tres décadas, por lo que se puede decir que la cantidad de casos a nivel mundial ha creceido de forma paralela al desarrollo industrial. De todos los casos, un $45 \%$ de ellos se presentan antes de los 6 meses, $60 \%$ en el primer año y $85 \%$ antes de los 5 años (7). La enfermedad suele ser un predecesor de otras enfermedades atópicas, ya que un $50 \%$ a $80 \%$ de las personas con DA desarrollan asma o rinitis alérgica posteriormente (8). 


\section{ETIOPATOGENIA}

La etiopatogenia de la dermatitis atópica es de carácter multifactorial, resultando de la interacción de factores genéticos, factores ambientales, defectos en la función barrera, susceptibilidad a infecciones cutáneas y una serie de factores inmunológicos.

En la DA se produce una respuesta de linfocitos de tipo Th2, determinada por factores genéticos y ambientales. Se ha demostrado que los factores genéticos tienen un claro papel en el desarrollo de la DA. Aproximadamente el $70 \%$ de los pacientes tienen antecedentes familiares de atopia.

\section{DIAGNÓSTICO}

El diagnóostico de la dermatitis atópica se realiza basado en la anamnesis, los síntomas y signos clínicos. La morfologíia y distribucion de las lesiones en piel pueden ser de gran ayuda. Sin embargo, no se dispone de ningún dato analítico o histopatológico que sea exclusivo de la enfermedad, por lo cual, se deberá tomar en consideración la historia clínica y el examen físico del paciente, así como el curso de la enfermedad para hacer el diagnóstico (7). Los niveles de lgE no son usados en la evaluación de rutina de los pacientes con DA (no todos los pacientes con criterios clínicos de DA presentan sensibilización IgE-mediada) (8). El tipo y la ubicación de las lesiones cutáneas varían con la edad del paciente y el curso y la duración de la enfermedad. En niños menores de 2 años ocurre comúnmente en las mejillas, en niños entre 2 y 12 años se presenta con mayor frecuencia en las zonas flexoras (codo y rodilla, cuello y muñeca), mientras que la DA en adolescentes $y$ adultos, las lesiones tienden a predominar en cara, manos, pies y superficies flexoras.

Se han propuesto diferentes criterios para la ayuda del diagnóstico, los más utilizados en la actualidad son los del grupo de trabajo del Reino Unido que establecen: (9)

Como criterio mandatorio la evidencia de prurito en piel: lesiones cutaneas pruriginosas (incluido referencia de padres de rascado).

Además de presentar 3 o máas de los siguientes hallazgos:

-Historia de afectacióon de los pliegues cutaneos: fosa antecubital, fosa poplíteas, cara lateral de los tobillos cuello y zona periorbitaria (pliegue de Dennie Morgan)

- Antecedente de asma o rinitis alergica (o historia de enfermedad atópica en un familiar de primer grado en un paciente menor de 4 años)

- Xerosis generalizada en el úultimo año.

- Inicio antes de los 2 años de vida (no se ultiliza en pacientes menores de 4 años)

-Dermatitis flexural visible (En menores de 4 años incluye dermatitis de mejillas, frente, cara externa de las extremidades)

Los criterios de Hanifin y Rajka (publicados en 1980 y modificados en 1997), para la ayuda del diagnóstico dividiendolo en criterios mayores y criterios menores, los cuales se presentan a continuación (10-11):

\section{Criterios mayores}

1. Prurito

2. Dermatitis crónica y recurrente

3. Morfología y distribución típica

4. Historia familiar o personal de atopia

\section{Criterios menores}

1. Xerosis.

2. Ictiosis, hiperlinearidad palmar o queratosis pilar.

3. Reactividad a pruebas cutáneas inmediata (tipo 1).

4. IgE sérico elevado.

5. Comienzo a temprana edad.

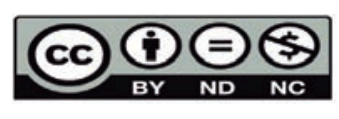


6. Tendencia a infecciones cutáneas (especialmente $\mathrm{S}$. aureus y herpes simple) o defecto en la inmunidad mediada por células.

7. Tendencia a dermatitis inespecífica en manos y pies.

8. Eccema del pezón.

9. Queilitis.

10. Conjuntivitis recurrente.

11. Pliegue infraorbitario de Dennie-Morgan.

12. Queratocono.

13. Catarata anterior subcapsular.

14. Oscurecimiento orbitario (ojeras).

15. Eritema o palidez facial.

16. Pitiriasis Alba.

17. Pliegues anteriores del cuello.

18. Prurito al sudar (hipersudoración).

19. Intolerancia a lanas y solventes orgánicos.

20. Acentuación perifolicular.

21. Intolerancia a alimentos.

22. Curso influenciado por factores ambientales o emocionales.

23. Dermografismo blanco.

Cabe destacar que a pesar de tener distintas herramientas como lo son criterios diagnósticos, no necesariamente deben de estar presentes en su totalidad, estos son un complemento y ayudan a orientar acerca de la patología.

\section{TRATAMIENTO}

Actualmente, no existe un tratamiento curativo para la dermatitis atópica, por lo que los tratamientos están orientados a prevenir la inflamación, las infecciones y al alivio sintomático de la enfermedad. Los fundamentos del tratamiento de la DA se basan en una combinación del cuidado adecuado de la piel, el uso diario de emolientes y el tratamiento antiinflamatorio, evitando el contacto con alérgenos e irritantes provocadores (12).
Los dermocorticoides tópicos son el tratamiento de primera línea. Su elección dependerá de la localización de las lesiones, tipo de eczema y grado de afectación. Si las lesiones son en cara, cuello o pliegues, se sugiere el uso de corticoides de baja potencia; si las lesiones son agudas y exudativas, se debe utilizar corticoides de potencia media y excipientes poco grasos, como las emulsiones o las cremas, y en eczemas de tipo crónico se emplea corticoides potentes y excipientes más grasos, como las pomadas o los ungüentos $(9,12-16)$.

El tacrolimús puede prescribirse como tratamiento de segunda línea. La fototerapia se usa bastante en los adultos, pero rara vez en los niños. Los tratamientos sistémicos, (ciclosporina, otros inmunosupresores y terapias dirigidas), son excepcionales y la mayoría de las veces deben ser prescritos por equipos especializados (17).

Recomendaciones para el cuidado de la piel en pacientes con DA(18):

- Educar y explicar al paciente y a sus familiares las características de la enfermedad.

-Factores específicos: dieta, aeroalergenos, contactantes.

- Evitar irritantes (productos químicos, perfumes, suavizantes, lejía, ambientador, humo de cigarro, etc)

-Baño con agua templada. No más de 5 a 10 minutos. Usar gel de baño de $\mathrm{pH}$ neutro o ácido.

- Tras el baño se debe secar al paciente con una toalla suave, evitando la fricción.

- Aplicar el emoliente inmediatamente 
después del baño, en toda la piel (piel húmeda) - Uso de emolientes diario. Si es necesario dos veces al día (disminuye el prurito y las necesidades de tratamiento con esteroides tópicos).

- El sol y la humedad resultan beneficiosos. Evitar el calor y la sudoración.

- Usar ropa de algodón. Evitar ropas de lana, fibras sintéticas, ropa apretada.

- Cortar las uñas y colocar guantes por la noche para evitar el rascado.

- Evitar el estrés.

- Reconocer los factores desencadenantes.

\section{CONCLUSIONES}

El efecto sobre la calidad de vida de los pacientes en edad adulta y de su familia es considerable y está subestimado.

La atención médica global de un paciente con DA y de su familia no solo debe incluir una educación terapéutica, sino que también debe ser planificada.

Las presentaciones atípicas de dermatitis atópica en adultos representan un desafío clínico.

Se recomienda educar al paciente no solo sobre la enfermedad, sino sobre la importancia de identificar posibles irritantes usados en el día a día, así como el adecuado manejo de estresantes emocionales.

\section{BIBLIOGRAFÍAS}

1. Weidinger S, Novak N. Atopic dermatitis.Lancet.2016;387:1109-22

2. Pautt-Lara E, Cala-Castro L, Harris $O$, et al. Dermatitis atópica de inicio tardío con patrón numular. Rev Alergia México. 2019; 66(4):483-487.

3. de Bruin Weller MS, Rockmann H, Knulst AC, Bruijnzeel-Koomen CA. Evaluation of the adult patient with atopic dermatitis. Clin Exp Allergy. 2013 Mar;43(3):279-91.

4. Brunner PM, Silverberg JI, Guttman-Yassky E, et al. Increasing comorbidities suggest that atopic dermatitis is a systemic disorder. J Invest Dermatol. 2016; 137:18-25.

5. Fölster-Holst R. Management of atopic dermatitis: are there differences between children and adults? J Eur Acad Dermatol Venereol. 2014;28 Suppl 3:5-8.

6. Farreras, Rozman. Medicina Interna. Dermatitis Atopica. Edicion 17

7. Rivero J. Dermatitis atópica. Revista medica de costa rica y centroamerica LXXIII 2016: (620) 711 - 716 .

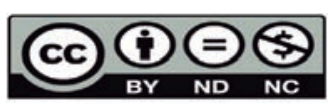


8. Vestergaard C, Deleuran M. Advances in the Diagnosis and Therapeutic Management of Atopic Dermatitis. Drugs. 2014;74:757-69.

9. William L Weston, MD, William Howe, MD Dermatitis atópica. (eczema) Patogénesis, manifestaciones clínicas, y diagnóstico. Uptodate, actualizado 30 mayo 2020.

10. Dammak A, Guillet S. Dermatitis atópica del niño. EMC-Tratado de Medicina [Internet]. 2015.

11. Cárdenas Medina A. Dermatitis atópica: los 5 puntos clave para su diagnóstico y manejo. España: Edicus; 2017.

12. Vestergaard C, Deleuran M. Advances in the Diagnosis and Therapeutic Management of Atopic Dermatitis. Drugs. 2014; 74:757-69.

13. Nowicki, Roman; et al. Atopic dermatitis: current treatment guidelines. Statement of the experts of the Dermatological Section, Polish Society of Allergology, and the Allergology Section, Polish Society of Dermatology. Postepy Dermatol Alergol. 2015 Aug; 32(4): 239-249

14. Sathishkumar D, Moss C. Topical Therapy in Atopic Dermatitis in Children. Indian J Dermatol. 2016;61(6). Citado en PubMed: PMID: 27904185.

15. Eichenfield LF, Tom WL, Berger TG, et al. Guidelines of Care for the Management of Atopic Dermatitis. Section 2. Management and Treatment of Atopic Dermatitis with Topical Therapies. J Am Acad Dermatol. 2014; 71:116-32.

16. Tollefson MM, Bruckner AL. Section on Dermatology. Atopic Dermatitis: Skin-Directed Management. Pediatrics. 2014; 134:1735-44.

17. Chong M, Fonacier L. Treatment of Eczema: Corticosteroids and Beyond. Clin Rev Allergy Immunol. 2016; 51:249-62.

18. S. Barbarot, H. Aubert, C. Bernier, et al. Dermatitis Atópica. ScienceDirect. 2016;50(4):1-22.

19. Ricardo Alonso Orayne Ekatherina, Rodríguez Sánchez María Beatriz, Hernández Fernández Mariela, Alonso González Maritza. Aspectos de interés sobre dermatitis atópica, su diagnóstico y tratamiento. Rev.Med.Electrón. 2019 ;41(2): 496-507. 